\title{
INFLUENCE OF D-GLUCOSE ON LIPID SOLID SUPPORT MEMBRANE SYSTEM AS ATTEMPT FOR BIOSENSING OF MEDICALLY RELEVANT MOLECULES
}

\author{
Galina Laputková, Michal Legiñ, Ján Sabo
}

\author{
P. J. Šafárik University, Faculty of Medicine, Slovak Republic: Department of Medical Biophysics
}

Summary: The influence of D-glucose on a lipid solid support system with the aid of impedance spectrocopy as a preliminary attempt for the biosensing of medical relevant molecules was studied. In spite of some shortcomings, s-BLM's proved to be an appropriate model for the study of lipid membrane - D-glucose interactions. The shortcomings were the roughness of the metal support, and the lack of homogeneity in the monolayer or multilayer lipid structures.

Key words: Supported lipid membrane; Biosensor; Glucose

\section{Introduction}

In recent years, so-called supported lipid membranes (s-BLM's), representing functional interfaces between biomaterial and the electrically conductive support - freshly cut tip of stainless steel, silver or platinum wire - have become a focus of intensive investigation and a frequently used model of cell membrane. S-BLM's, i.e. freely suspended lipid membranes on a hydrophilic support, have been developed by Tien and Salamon (10). The system overcomes some of the drawbacks of planar black lipid membranes. It is also noted for its long term stability and its ease of preparation in a reproducible way. Supported lipid membranes have been used in fundamental studies of lipid assemblies, in studies of membrane structure and membrane dynamics, and in studies of the electrochemical properties of membranes $(1,8)$. However, the primary attraction of s-BLM's is their possible application to the field of biosensing. Their applications include providing the means to observe changes in the properties of a deposited lipid layer induced by the layer's surroundings (3). The unique features of s-BLM's are the prerequisite for incorporation of various biological molecules, such as poreforming peptides, channel proteings, and receptors. Such molecules retain their native functions after incorporation, thus allowing the interactions of incorporated molecules with external ligands (9).

The topic of this paper is to establish the influence of D-glucose on s-BLM with the aid of impedance spectroscopy as a preliminary attempt for the biosensing of medical relevant molecules. The choice of D-glucose was influenced especially by the fact that D-glucose changes the electrical properties of s-BLM modified with anthraquinone-2-sul- phonic acid as we demonstrated with cyclic voltammetry in our previous work (5).

\section{Materials}

1,2-Diacyl-sn-glycero-3-phosphocholine (DPPC) from egg yolk was purchased from Sigma. N-dodecane (Sigma), D-glucose, and all chemical compounds were of analytical grade and were used without further purification. The solutions of inorganic salts were prepared in freshly distilled and deionized water obtained from a Millipore filter system. Deionized water had a resistivity of $\sim 18.2 \mathrm{M} \Omega \mathrm{cm}$.

\section{Formation of supported lipid bilayers}

The simplest suitable technique for the formation of s-BLM's has proven to be the deposition of a lipid membrane on a freshly created metallic surface (10). The formation of s-BLM is based on the interaction of amphiphilic lipid molecule with a nascent metal surface. Prior to the self-assembling of the lipid bilayer on the platinum support, a Teflon coated platinum wire $(0.5 \mathrm{~mm}$ diameter $)$ was immersed in a lipid forming solution, and, while still immersed, the wire was cut so as to provide a sufficiently reproducible section of the wire. The membrane forming solution used in the experiments was prepared by mixing the ethanol-lipid solution with n-dodecane to attain a final lipid concentration of $5 \%$. A drop of the solution will adhere to the electrode tip, because of the great affinity between the lipid molecules and the freshly cut metal surface. The electrode tip, with the drop of lipid solution adhered to it, was then immersed into the aqueous electrolyte bath, which preferably consists of $100 \mathrm{mmol}^{-1} \mathrm{KCl}^{\mathrm{KC}}$ at a $\mathrm{pH}$ of 5.5 . 


\section{Impedance measurements}

The electrochemical properties of the platinum surface were studied by impedance spectroscopy. The electrical impedance $Z(\omega)$ is complex, and determined by the following formula:

$$
Z(\omega)=\frac{U(\omega)}{I(\omega)}=|Z(\omega)| \cdot e^{i \varphi(\omega)}
$$

where $U(\omega)$ is the voltage applied to the system, $I(\omega)$ is the measured current response, and $\omega$ is the angular frequency.

For data representation, we used the polar coordinates of $Z(\omega)$, the absolute value $|Z(\omega)|$, and the phase $\varphi(\omega)$.

For the impedance spectroscopy experiments, we used the commercially available electrochemical analyzer Zahner Elektrik IM6e (Zahner Meßtechnik, GmbH, Germany), and controlled it with a personal computer. The measuring cell contained the conventional three-electrode configura- tion. The Teflon-covered platinum wire electrode supporting the membrane served as a working electrode. An identical Teflon-coated platinum wire was used as the counter electrode. The reference electrode $\mathrm{Ag} / \mathrm{AgCl}$ (saturated $\mathrm{KCl}$ ) was isolated from the cell by an agar bridge. The measuring cell was surrounded by a water jacket to maintain stable temperature, and the cell with jacket was then placed into a Faraday cage. All experiments were carried out at room temperature $(21.0 \pm 0.2)^{\circ} \mathrm{C}$.

The EIS experiments were performed at a frequency range of $100 \mathrm{mHz}-10 \mathrm{kHz}$, at 15 steps per decade. Impedance was measured with an applied bias voltage $0 \mathrm{mV}$, at sinusoidal excitation amplitude of $50 \mathrm{mV}$. The measurement was performed 10 times at each frequency, and averaged during the run. The smoothed EIS data were analyzed using complex non-linear regression least square (CNRLS) fit to a model represented by an equivalent electrical cir-

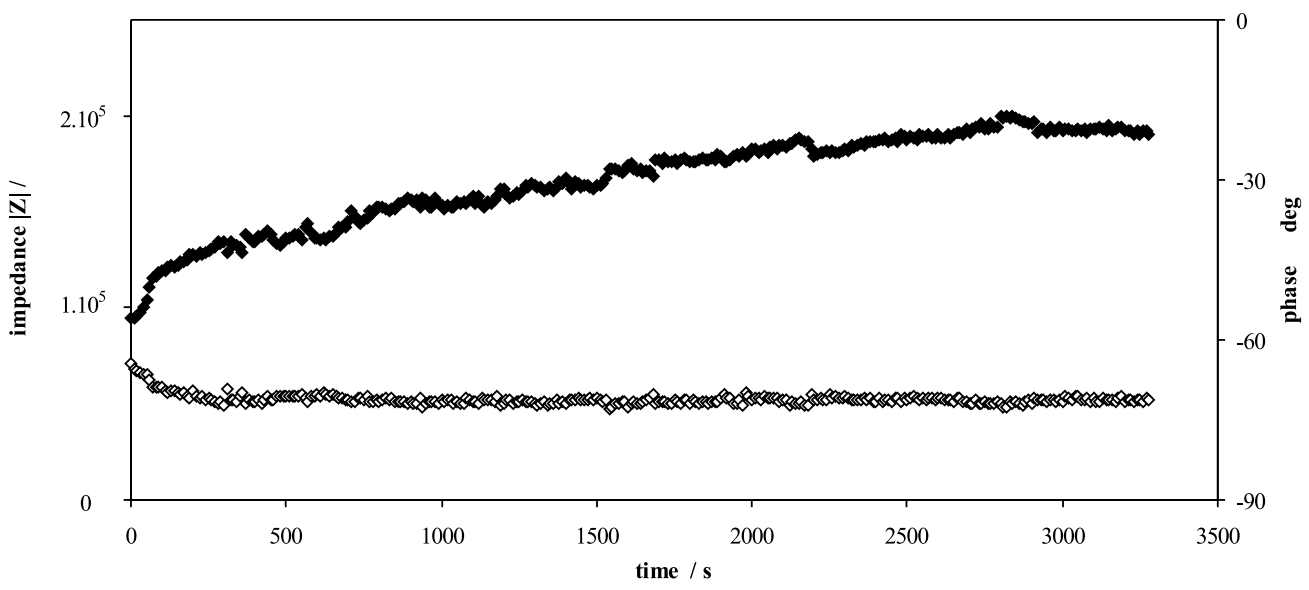

Fig. 1: The time variation of impedance $|\mathrm{Z}|(\diamond)$ and phase shift $\varphi(\diamond)$ of s-BLM developing on platinum support in 100 mmol. $\mathrm{l}^{-1} \mathrm{KCl}$, the amplitude of the applied a.c. signal $\mathrm{U}=50 \mathrm{mV}, \mathrm{f}=10 \mathrm{kHz}, \mathrm{U}_{\text {bias }}=0 \mathrm{mV}$.

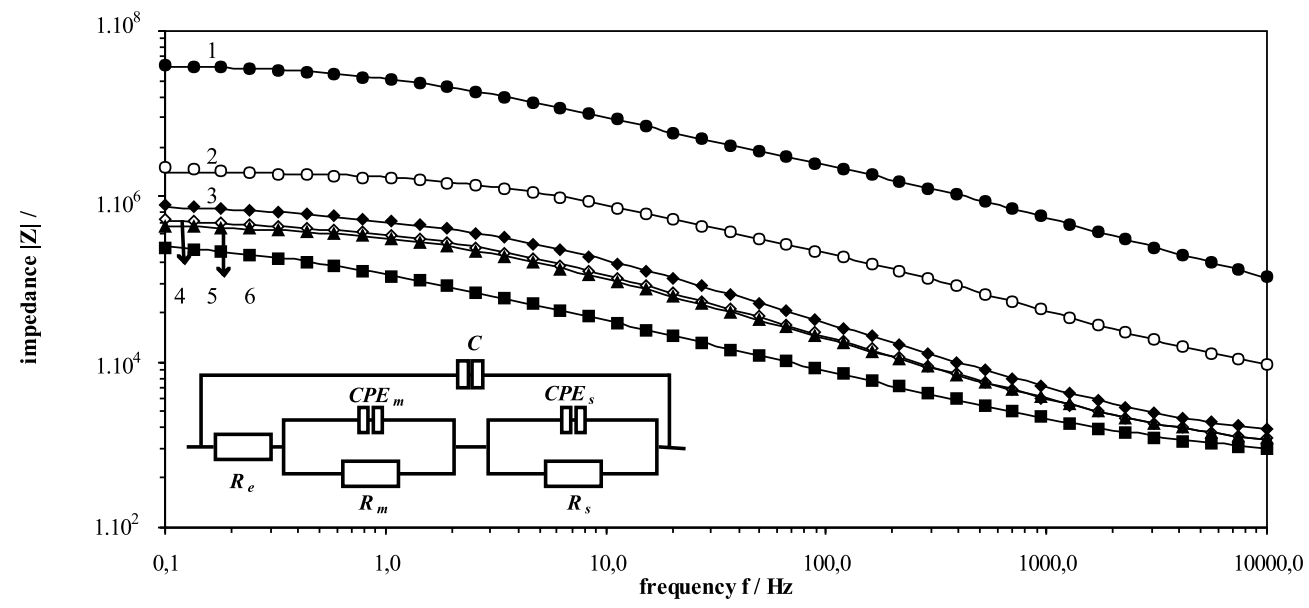

Fig. 2: Effect of D-glucose on the plots of impedance $|\mathrm{Z}|$ of s-BLM on platinum in $100 \mathrm{mmol}^{-1} \mathrm{KCl}^{-1} 1 . \mathrm{s}-\mathrm{BLM}, 0 \mathrm{mmol.1}$ ${ }^{1}$ glucose, 2. s-BLM, 3 mmol..$^{-1}$ glucose, 3. s-BLM, 5 mmol..$^{-1}$ glucose, 4. s-BLM, 10 mmol.1.$^{-1}$ glucose, 5. s-BLM, 50 mmol.1 ${ }^{1}$ glucose, 6 . bare platinum, $0 \mathrm{mmol} \cdot \mathrm{l}^{-1}$ glucose. The amplitude of the applied a.c. signal $\mathrm{U}=50 \mathrm{mV}, \mathrm{U}_{\text {bias }}=0 \mathrm{mV}$. (-) fit to the equivalent circuit model using CNRLS regression. Inset: An equivalent circuit for metal-supported lipid membrane. 
cuit. The software used for data analysis was a commercial program from Zahner Elektrik.

\section{Results and discussion}

Fig. 1 shows the typical time course of the impedance and phase angle during the developing of the lipid bilayer on the support. Both the impedance values and the phase values were constant, and both values also corresponded to the steady state of the membrane. Stable membrane structures were in most cases reached within an hour. After stability was reached, it was long lastingly maintained under the unchanging external conditions. For most of the s-BLM's, membrane degradation was not observed during a time interval of 24 hours.

Fig. 2 (curve 1.) shows AC impedance spectroscopy of an s-BLM that was immersed in $100 \mathrm{mmol.l}^{-1} \mathrm{KCl}$ for approximately 2 hours. For the data analysis we chose a simple electrical equivalent circuit (in the inset of Fig. 2). The model describes the most important properties of a lipid membrane which is supported on metal. The electrical circuit consists of the resistance $R_{e}$, which corresponds to the ohmic behavior of the electrolyte. The parallel arrangement of the resistance $R_{s}$ and the constant phase element $\mathrm{CPE}_{\mathrm{s}}$ represents the contribution of the electrode - electrolyte interface. The parallel arrangement of the resistance $R_{m}$ and a constant phase element $\mathrm{CPE}_{\mathrm{m}}$ represents the impedance of the lipid film at the interface between the electrolyte and platinum. $\mathrm{C}_{\mathrm{c}}$ is the stray capacitance of the measuring cell.

The Nyquist plot of the measured impedance spectrum of s-BLM on the metal (not shown) corresponds with the suppressed arc. The arc can be formally described by introduction of a constant phase element (CPE) instead of the capacitance. The appearance of the suppressed arc indicates a distribution of relaxation times of the circuit. There are two possible explanations for the appearance of the arc. One explanation attributes it to the properties of the metal electrode supporting the membrane, especially to the electrode's porousness and rough surface (6). The other explanation is that it reflects the imperfect organization of the lipid bilayer on the electrode's rough surface.

To estimate the fractional coverage of the electrode, it is useful to employ Nagel and Scott's theory (7). By assuming that ion transport occurs only at the defect sites of the lipid layer, it is reasonable to express the fractional coverage of the electrode as: $\theta=1-\left(R_{S}^{\text {bare }} / R_{m}\right)$, where $R_{s}^{\text {bare }}=0.65$ $\mathrm{k} \Omega . \mathrm{cm}^{2}$ represents the bare electrode - electrolyte resistance obtained by fitting the impedance spectrum of bare platinum to the equivalent circuit, and where $\mathrm{R}_{\mathrm{m}}$ corresponds to the membrane resistance.

The table compares the s-BLM lipid coverage values with the glucose treated lipid membranes values.

As a preliminary check of membrane quality for biosensing, the membrane - D-glucose interactions were studied. The membrane parameters were followed using impedance spectroscopy. Fig. 2 exhibits the impedance spectra of supported lipid bilayer in $100 \mathrm{mmol}^{-1} \mathrm{KCl}$ before and after the addition of D-glucose. The same membrane was used for all measurements by continuous increasing of glucose concentration. It can be seen from the spectra, that glucose led to significantly change the membrane parameters. CNRLS regression results of s-BLM and s-BLM treated with $3 \mathrm{mmol}^{-1} \mathrm{D}$-glucose is given in the Table. At a concentration of $3 \mathrm{mmol}^{-\mathrm{I}^{-1}}$, the membrane resistance drops to a value $3.90 \mathrm{k} \Omega . \mathrm{cm}^{2}$, significantly decreases the fractional coverage of the electrode and consequently the ion permeability of the lipid layer increase. The continuous increasing of glucose leads to the total destruction of the lipid membrane. The phospholipid portion of s-BLM is not sufficiently flexible to "withstand" the perturbing molecules. The electrical resistance and the capacitance of the membrane are significantly affected by the glucose and the structure of the s-BLM was disrupted for the concentration of glucose exceeding $10 \mathrm{mmol}^{-1}$.

\section{Conclusions}

In spite of some shortcomings, s-BLM's proved to be an appropriate model for the study of lipid membrane - D-glucose interactions. The shortcomings were the roughness of the metal support, and the lack of homogeneity in the monolayer or multilayer lipid structures.

We found evidence that glucose influences the properties of s-BLM's in physiologically relevant concentrations. But we also observed that the influence of the glucose is different if s-BLM's are modified with anthraquinone-2-sulphonic acid [4], where the membrane current was reduced

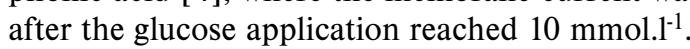

The results nevertheless support suggestions (2) that sugars, either free in solution or covalently linked to membrane constituents, can affect the properties and stability of bilayers. The results also indicate that glucose initiated

Tab. 1: CNRLS fitting results.

\begin{tabular}{|l|c|c|c|c|c|c|c|}
\hline & \multirow{2}{*}{$\mathrm{R}_{\mathrm{m}} / \Omega$} & \multirow{2}{*}{$\mathrm{Rm} / \mathrm{k} \Omega . \mathrm{cm}^{2}$} & \multicolumn{2}{|c|}{$\mathrm{CPE}_{\mathrm{m}}$} & \multirow{2}{*}{$\mathrm{C}_{\mathrm{m}} / \mu \mathrm{F}_{\mathrm{n}} \mathrm{cm}^{-2}$} & $\mathrm{~d} / \mathrm{nm}$ & $\theta$ \\
\cline { 4 - 8 } & & & $\mathrm{V}=\mathrm{C}_{\mathrm{m}}(\mathrm{x} / \mathrm{d}) / \mathrm{pF}$ & $\alpha=1-(\mathrm{x} / \mathrm{d})$ & & \\
\hline s-BLM & 39.70 & 78.01 & 439 & 0.711 & 0.77 & 2.4 & 0.9916 \\
\hline s-BLM + 3mmol.1. ${ }^{-1}$ glucose & 1.99 & 3.90 & 4970 & 0.736 & 9.58 & 0.2 & 0.8328 \\
\hline
\end{tabular}

${ }^{*} \alpha$ - approximation of penetration depth $\mathrm{x}$ of conduction into an insulating surface layer of thickness $\mathrm{d}$;

$\mathrm{Z}_{\mathrm{CPE}}=1 /\left(\omega_{0} \mathrm{~V}\left(\mathrm{j} \omega / \omega_{0}\right)=2 \pi .1000\right.$. 
changes of the impedance characteristics of s-BLM's make it reasonable to use s-BLM's for biosensing.

\section{Acknowledgement}

This work was supported by internal grant 26/20022005 from the Faculty of Medicine of P. J. Safarik University, Kosice, Slovak Republic.

\section{References}

1. Bordi F, Cametti C, Gliozzi A. Impedance measurements of self-assembled lipid b layer membranes on the tip of an electrode. Bioelectrochemistry 2002;57:39-46.

2. Crowe JH, Crowe LM, Carpenter JF et al. Interaction of sugars with membranes. Biochim Biophys Acta 1988;947:367-84.

3. Han X, Wang E. Ion-channel sensing of ferricyanide anion based on a supported bilayer lipid membrane. Anal Sci 2001;17:1171-4.

4. Laputková G, Sabo J. Cyclic voltammetry study of glucose and insulin interactions with supported lipid membrane. Bioelectrochemistry 2002;56:185-8.

5. Laputková G, Sabo J. Effect of increased glucose concentration on the supported bilayer lipid membrane. Transactions of the Universities of Košice 2000;58:42-6
6. MacDonald JR. Impedance spectroscopy. New York: John Wiley and Sons, 1987:346

7. Nagle JF, Scott HL. Lateral compressibility of lipid mono- and bilayers: Theory of membrane permeability. Biochim Biophys Acta 1978;513,236-43.

8. Sabo J, Ottova A, Laputkova G, Legin M, Vojcikova L, Tien HT. A combined AC DC method for investigation supported lipid membranes. Thin Solid Films 1997;306:112-8

9. Salamon Z, Tollin G. Surface plasmon resonance studies of complex formation between cytochrome $\mathrm{c}$ and bovine cytochrom $\mathrm{c}$ oxidase incorporated into a planar lipid bilayer. II Binding of cytochrome $\mathrm{c}$ to oxidase-containing cardiolipin/ phosphatidylcholine membranes. Biophys J 1996;71:858-67.

10. Tien HT, Salamon Z. Formation of self-assembled lipid bilayers on solid substrates. Bioelectroch. Bioener 1989;99:211-8.

Galina Laputková, Faculty of Medicine, Department of Biophysics, Tr. SNP 1, 04011 Košice, Slovak Republic. e-mail: laputk@medic.upjs.sk 